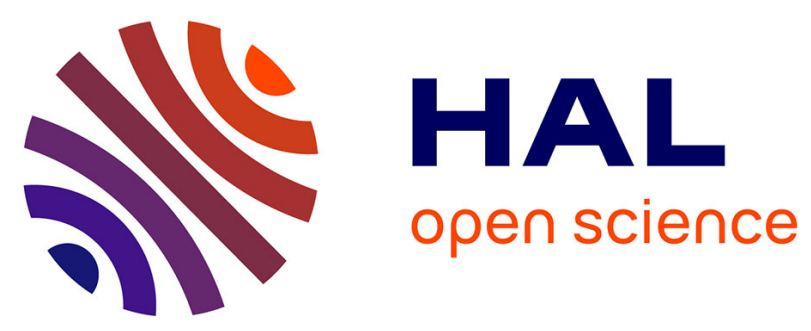

\title{
Calcul simultané de la polarisabilité et de l'énergie de transition électronique des molécules. Cas particulier de l'éthylène et du benzène - Deuxième Partie: Comparaison des incréments de réfractivité de la double liaison éthylénique et du cycle benzénique
}

\author{
J. Barriol, Jérémi Regnier
}

\section{To cite this version:}

J. Barriol, Jérémi Regnier. Calcul simultané de la polarisabilité et de l'énergie de transition électronique des molécules. Cas particulier de l'éthylène et du benzène - Deuxième Partie: Comparaison des incréments de réfractivité de la double liaison éthylénique et du cycle benzénique. J. Phys. Radium, 1955, 16 (8-9), pp.641-644. 10.1051/jphysrad:01955001608-9064100 . jpa-00235236

\author{
HAL Id: jpa-00235236 \\ https://hal.science/jpa-00235236
}

Submitted on 1 Jan 1955

HAL is a multi-disciplinary open access archive for the deposit and dissemination of scientific research documents, whether they are published or not. The documents may come from teaching and research institutions in France or abroad, or from public or private research centers.
L'archive ouverte pluridisciplinaire HAL, est destinée au dépôt et à la diffusion de documents scientifiques de niveau recherche, publiés ou non, émanant des établissements d'enseignement et de recherche français ou étrangers, des laboratoires publics ou privés. 


\title{
LE JOURNAL DE PHYSIQUE
}

\section{LE RADIUM}

\section{GALGUL SIMULTANÉ DE LA POLARISABILITÉ \\ ET DE L'ÉNERGIE DE TRANSITION ÉLEGTRONIOUE DES MOLÉCULES. GAS PARTIGULIER DE. L'ÉTHYLĖNE ET DU BENZĖNE}

\author{
Par J. BARRIOL et J. RÉGNIER,
} Faculté des Sciences de Nancy.

\begin{abstract}
Sommaire. - Selon la méthode'exposée dans la première partie, l'incrément dẻ polarisabilité de la double liaison éthylénique est calculé à l'aide de MO simples. Le résultat moins bon que celui déjà obtenu à partir d'une fonction d'onde plus élaborée, peut toutefois être utilement comparé à l'incrément dâ aux doubles liaisons du cycle benzénique. On montre ainsi que l'incrément du cycle benzénique (qui vaut trois fois l'incrément de double liaison éthylénique) est dû aux seules doubles liaisons.

Dans un second paragraphe l'identification de deux valeurs de la polarisabilité longitudinale, obtenues de façon différente, permet d'arriver à une évaluation de la longueur d'onde de la transition parallèle dans $\mathrm{C}_{2} \mathrm{H}_{4}$. La formule, qui rappelle celle obtenue à partir du modèle métallique, est toutefois en bien meilleur accord avec les faits.
\end{abstract}

\section{Deuxième Partie :}

Comparaison des incréments de réfractivité de la double liaison éthylénique et du cycle benzénique ( $\left.{ }^{1}\right)$.

La méthode générale donnée dans la première partie sera encore employée ici, la seule différence étant le choix de la fonction d'onde $\psi_{0}$ représentant l'état fondamental. Pour la simplicité des calculs, on prendra pour le benzène la MO antisymétrisée sans faire intervenir les formes ioniques, ou ce qui revient au même, sans tenir compte des interactions de configurations. Ceci exige alors que la fonction d'onde de l'état fondamental de $\mathrm{C}_{2} \mathrm{H}_{4}$ soit une $\mathrm{MO}$ simple sans correction d'état ionique.

Éthylène. - Les notations sont les mêmes que dans la première partie, mais cette fois la distribution des électrons $\pi$ est donnée dans l'état fondamental par

$$
\psi_{0}=\frac{[a(\mathrm{I})+b(\mathrm{I})][a(2)+b(2)]}{2(\mathrm{I}+S)} .
$$

Le calcul de la polarisabilité suivant $\mathrm{Oy}$ se ramène à celui de

$$
K_{3}^{2}=\int\left(y_{1}+y_{2}\right)^{2} \psi_{0}^{2} \mathrm{~d} \tau_{1} \mathrm{~d} \tau_{2}
$$

( ${ }^{1}$ Les renvois à la première partie seront désignés par la lettre $\mathrm{I}$.
Négligeant encore les intégrales du type

$$
\int y^{2} a b \mathrm{~d} \tau
$$

nous obtenons

$$
K_{i}^{\prime}=\frac{R^{2}+4 C_{Y}^{2}}{2(\mathrm{I}+S)}
$$

ce qui conduit à l'aide de (5.1) à

$$
\alpha_{j}=\frac{\left(R^{2}+4 C_{1}^{2}\right)^{2}}{2(\mathrm{I}+S)^{2} a_{0}} .
$$

De la même manière la polarisabilité suivant $\mathrm{O} x$ s'évalue à partir de

$$
\mathbf{h}_{x}^{2}=\frac{2 C_{x}^{2}}{\mathrm{I}+S} .
$$

Pour l'axe $\mathrm{O} z$ il s'introduit encore l'intégrale

$$
\int z^{2} a b d \tau
$$

que l'on ne peut plus négliger mais que l'on ramène à

$$
\zeta^{2} \int a b \mathrm{~d} \tau=\zeta^{2} S
$$

Le JOURNAL DE PHYSIQUe ET Le RADIUM. - T. 16. - $\mathbf{N}^{\text {os }} 8-9$. - AOUT-SEPTEMBRE 1955. 
où $S$ est l'intégrale de superposition et $\zeta$ la cote $z$ donnant le chevauchement maximum. Ainsi

$$
K_{z}^{2}=\frac{2\left(C_{z}^{2}+\zeta^{2} S\right)}{\mathrm{I}+S} .
$$

La polarisabilité moyenne

s'écrit alors

$$
\bar{\alpha}=\frac{\alpha_{x}+\alpha_{y}+\alpha_{z}}{3}
$$

$$
\bar{\alpha}=\frac{\mathrm{I}}{6(\mathrm{I}+S)^{2} \alpha_{0}}\left[\left(R^{2}+4 C_{\zeta}^{2}\right)^{2}+16 C_{\imath}^{1}+\mathrm{I} 6\left(C_{\bar{z}}^{2}+\zeta^{2} S\right)^{2}\right],
$$

les grandeurs $C_{1}, C_{1}, C_{\approx}$ et $\zeta$ ayant les mêmes valeurs que dans $I$.

L'expression définitive de $\alpha$

$$
\begin{aligned}
\bar{\alpha}=\frac{\mathrm{I}}{6(\mathrm{I}+S)^{2} a_{0}}[ & \left(R^{2}+24 \frac{a_{0}^{2}}{Z^{2}}\right)^{2}+\left(24 \frac{a_{0}^{2}}{Z^{2}}\right)^{2} \\
& \left.+\left(8 \frac{a_{0}^{2}}{Z^{2}}(9+S)+4 \frac{a_{0}}{Z} R\right)^{2}\right]
\end{aligned}
$$

conduit pour incrément de double liaison éthylénique

$$
P=\frac{4 \pi}{3} N \bar{\alpha}
$$

à la valeur $P=5,05$, nettement moins bonne que la valeur expérimentale 2,95 .

Il est hors de doute que cette divergence doit être attribuée au fait que la fonction MO introduit une contribution trop grande des états ioniques. Ne tenant aucun compte de la corrélation électronique, elle fait que le champ appliqué accumule les électrons en bout de chaîne et explique ainsi la prédominance du terme $\alpha_{3}$.

Benzène. - Avec les notations de la figure 1,

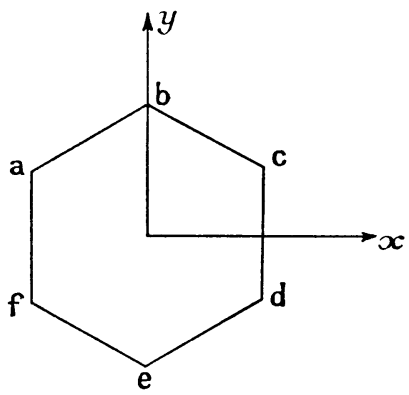

Fig. I.

la distribution des électrons $\pi$ dans l'état fondamental s'obtiendra à partir des MO occupées

$$
\begin{aligned}
& \Phi_{A}=A(a+b+c+d+e+f), \\
& \Phi_{B}=B(a+2 b+c-d-2 e-f), \\
& \Phi_{C}=C(a-c-d+f) .
\end{aligned}
$$

en construisant la fonction d'onde antisymétrique $\Theta=\frac{\mathrm{I}}{\sqrt{6 !}} \mathcal{Q} \Phi_{A}(\mathrm{I}) \alpha(\mathrm{I}) \Phi_{A}(2) \beta(2) \ldots \Phi_{C}(5) \alpha(5) \Phi_{C}(6) \beta(6)$, où $A, B, C$ sont des constantes de normation et $\alpha$ et $\beta$ les fonctions de spin.

Ceci posé, le calcul de la polarisabilité le long de l'axe $\mathrm{Oy}$ revient là encore à l'évaluation de

$$
K_{y}^{2}=\int\left(y_{1}+y_{2}+\ldots+y_{6}\right)^{2} \boldsymbol{\theta}^{2} \mathrm{~d} \tau_{1} \ldots \mathrm{d} \tau_{6} .
$$

Une transformation classique permet d'écrire

$$
\begin{aligned}
\Lambda_{1}= & \int\left(y_{1}+y_{2}+\ldots+y_{6}\right)^{2} \Phi_{A}(\mathrm{I}) \alpha(\mathrm{I}) \ldots \\
& \times \Phi_{C}(6) \beta(6) \mathfrak{Q} \Phi_{A}(\mathrm{I}) \alpha(\mathrm{I}) \ldots \Phi_{C}(6) \beta(6) \mathrm{d} \tau_{1} \ldots \mathrm{d} \tau_{6} .
\end{aligned}
$$

Or les fonctions $\Phi_{\Lambda}, \Phi_{B}$ et $\Phi_{C}$ étant orthogonales, l'expression de $\mathrm{K}_{r}^{2}$ se simplifie et devient

$$
\begin{array}{r}
K_{\jmath}^{2}=2\left[\int y^{2} \Phi_{A}^{2} \mathrm{~d} \tau+\int y^{2} \Phi_{B}^{2} \mathrm{~d} \tau+\int y^{2} \Phi_{C}^{2} \mathrm{~d} \tau\right. \\
-2 \int y \Phi_{A} \Phi_{B} \mathrm{~d} \tau-2 \int y \Phi_{A} \Phi_{C} \mathrm{~d} \tau \\
\left.-2 \int y \Phi_{B} \Phi_{C} \mathrm{~d} \tau\right] .
\end{array}
$$

Chacune de ces intégrales s'effectue sans difficulté. Il vient ainsi

$$
\int y^{2} \Phi_{A}^{2} \mathrm{~d} \tau=A^{2}\left[3 R^{2}+\frac{9 R^{2} S}{2}+6 C_{j}^{2}\right],
$$

où

$$
C_{3}^{2}=\int y_{a}^{2} a^{2} \mathrm{~d} \tau,
$$

l'origine étant au centre de symétrie de la molécule.

La normation de $\boldsymbol{\Phi}_{\mathrm{A}}$ fournit

$$
6 A^{2}=\frac{\mathrm{I}}{\mathrm{I}+2 S},
$$

d'où

$$
\int y^{2} \Phi_{A}^{2} \mathrm{~d} \tau=\frac{R^{2}\left(\mathrm{I}+\frac{3 S}{2}\right)+2 C_{5}^{2}}{2(\mathrm{I}+2 S)} .
$$

On montrerait de même que

$$
\int y^{2} \Phi_{\bar{B}}^{2} \mathrm{~d} \tau=\frac{3 R^{2}}{4}+\frac{C_{3}^{2}}{\mathrm{I}+S}
$$

et que

$$
\int y^{2} \Phi_{C}^{2} \mathrm{~d} \tau=\frac{R^{2}+4 C_{3}^{2}}{4(\mathrm{I}+S)} .
$$


Enfin les termes rectangles donnent :

$$
\begin{gathered}
\int y \Phi_{A} \Phi_{B} \mathrm{~d} \tau=\frac{R^{2}(2+3 S)^{2}}{4(\mathrm{I}+2 S)(\mathrm{I}+S)}, \\
\int y \Phi_{A} \Phi_{C} \mathrm{~d} \tau=\int y \Phi_{B} \Phi_{C} \mathrm{~d} \tau=\mathrm{o},
\end{gathered}
$$

de sorte que

$$
K_{y}^{2}=\frac{R^{2}}{I+S}+2 C_{j}^{2}\left(\frac{\mathrm{I}}{\mathrm{I}+2 S}+\frac{2}{\mathrm{I}+S}\right) .
$$

Par raison de symétrie on a d'ailleurs :

$$
K_{\bar{\chi}}^{2}=K_{\jmath}^{2} .
$$

Il reste donc à évaluer :

$$
\begin{aligned}
K_{z}^{2}= & \int\left(z_{1}+\ldots+z_{6}\right)^{2} \Phi_{A}(\mathrm{I}) \alpha(\mathrm{I}) \ldots \\
& \times \Phi_{C}(6) \beta(6) \mathcal{Q} \Phi_{A}(\mathrm{I}) \alpha(\mathrm{I}) \ldots \Phi_{C}(6) \beta(6) \mathrm{d} \tau_{1} \ldots \mathrm{d} \tau_{6}
\end{aligned}
$$

qui se trouve être égal à

$$
\begin{aligned}
& 2\left[\int^{*} z^{2} \Phi_{A}^{2} \mathrm{~d} \tau+\int z^{2} \Phi_{B}^{2} \mathrm{~d} \tau+\int z^{2} \Phi_{C}^{2} \mathrm{~d} \tau\right. \\
& \left.\quad-2 \int z \Phi_{A} \Phi_{B} \mathrm{~d} \tau-2 \int z \Phi_{A} \Phi_{C} \mathrm{~d} \tau-2 \int z \Phi_{B} \Phi_{C} \mathrm{~d} \tau\right] .
\end{aligned}
$$

On trouve facilement

$$
\begin{aligned}
& \int z^{2} \Phi_{A}^{2} \mathrm{~d} \tau=\frac{C_{z}^{2}+2 \zeta^{2} S}{\mathrm{I}+2 S}, \\
& \int z^{2} \Phi_{B}^{2} \mathrm{~d} \tau=\frac{C_{z}^{2}+\zeta^{2} S}{\mathrm{I}+S}, \\
& \int z^{2} \Phi_{C}^{2} \mathrm{~d} \tau=\frac{C_{z}^{2}+\zeta^{2} S}{1+S},
\end{aligned}
$$

le terme $C_{\Xi}^{2}$ représente l'intégrale

$$
\int z^{2} a^{2} \mathrm{~d} \tau
$$

et

$$
\zeta^{2} S=\int z^{2} a b d \tau=\zeta^{2} \int a b a \tau
$$

$\zeta$ étant la valeur de $z$ donnant le maximum de $a b$. De plus

$$
\int z \Phi_{A} \Phi_{B} \mathrm{~d} \tau=\int z \Phi_{A} \Phi_{C} \mathrm{~d} \tau=\int z \Phi_{B} \Phi_{C} \mathrm{~d} \tau=0
$$

Ainsi

$$
\Lambda_{z}^{2}=2\left(\frac{C_{z}^{2}+2 \zeta^{2} S}{\mathrm{I}+2 S}+2 \frac{C_{z}^{2}+\zeta^{2} S}{\mathrm{I}+S}\right) .
$$

Écrivant alors que la polarisabilité moyenne

$$
\bar{\alpha}=\frac{\alpha_{x}+\alpha_{1}+\alpha_{z}}{3}=\frac{2 \alpha_{x}+\alpha_{z}}{3}
$$

est donnée par

$$
\begin{aligned}
\bar{\alpha} & =\frac{8 \pi^{2} m e^{2}}{9 h^{2}}\left(2 K_{\imath}^{4}+K_{z}^{1}\right) \\
& =\frac{2}{9 a_{0}}\left(2 K_{x}^{4}+K_{z}^{4}\right)
\end{aligned}
$$

il vient pour incrément de réfractivité molaire du cycle benzénique

$$
P=\frac{4 \pi}{3} N \bar{\alpha}=\mathrm{I} 6,5
$$

en prenant $R={ }_{1}, 39 \AA, Z=3,43$ et $S=0,232$. Or, la réfractivité moléculaire du cycle benzénique est, expérimentalement, égale à trois fois la réfractivité de la double liaison éthylénique. Le rapport des incréments calculés ici est de 3,25. Cependant, étant donné la grande sensibilité de $\alpha$ à la valeur de la charge d'écran, et la variation de la réfractivité des électrons $\sigma$ avec la distance internucléaire (les tables de Landolt donnent [2] $\left(G_{11}-C_{110}\right)-\left(C_{\mathrm{d} 11}-C_{\mathrm{d} 11}\right)=0,43$ pour $\bar{\alpha})$, l'accord peut être considéré comme satisfaisant.

On peut d'ailleurs remarquer qu'en introduisant dans la fonction d'onde du benzène les interactions de configurations, l'importance des termes $\alpha_{x}$ et $\alpha_{y}$ diminuerait beaucoup. Il est à penser que l'on retrouverait trois fois la valeur 2,8 I calculée dans le cas de $\mathrm{C}_{2} \mathrm{H}_{4}$, mais ce serait au prix d'une complication énorme des calculs.

Calcul approché de l'énergie de transition électronique dans l'éthylène. - Reprenons pour $\mathrm{C}_{2} \mathrm{H}_{4}$ Ia $\mathrm{MO}$ liante

$$
\varphi_{0}(\mathrm{I})=\frac{a(\mathrm{I})+b(\mathrm{I})}{\sqrt{2(\mathrm{I}+S)}}
$$

et rappelons que pour le calcul de $\alpha$, nous avons introduit une fonction $\psi_{2}$ telle que

$$
\boldsymbol{K}_{y} \Psi_{2}=y \Psi_{0} \text {. }
$$

Or on peut montrer que le produit scalaire

$$
\left(\frac{y \psi_{0}(\mathrm{I})}{K_{3}}, \frac{a(\mathrm{I})-b(\mathrm{I})}{\sqrt{2(\mathrm{I}-S)}}\right)
$$

est très sensiblement égal à $\mathbf{~}$. Ceci suggère le calcul de $\alpha_{1}$, donné par

$$
\sigma_{\gamma}=\frac{2 e^{2}\left(\int \psi_{2} y \psi_{0} \mathrm{~d} \tau\right)^{2}}{\boldsymbol{E}_{2}-\boldsymbol{E}_{0}}
$$

en prenant pour $\psi_{0}$ et $\psi_{2}$ les MO liante et antiliante respectivement. On trouve

$$
\alpha_{y}=\frac{e^{2} R^{2}}{\left(E_{2}-E_{0}\right)\left(\mathrm{I}-S^{2}\right)} .
$$


Admettant alors que, par suite de l'insensibilité de $\alpha$ au choix de la fonction d'onde, les valeurs de $\alpha_{3}$ données par (1.II) et (2.II) coïncident, il vient :

$$
E_{2}-E_{0}=\frac{h^{2}}{2 \pi^{2} m R^{2}}\left(\frac{R^{2}}{R^{2}+4 C_{1}^{2}}\right)^{2} \frac{\mathrm{I}+S}{\mathrm{I}-S} .
$$

On notera l'analogie de cette formule avec celle obtenue à partir du modèle métallique. La longueur d'onde calculée

$$
\lambda=\frac{h c}{E_{2}-E_{0}}=\mathrm{I} 560 \AA
$$

correspond approximativement à celle de la transition parallèle dans l'éthylène [3], cette approximation étant d'ailleurs meilleure dans le cas des chaînes courtes que celle donnée par le modèle de l'électron libre.

\section{BIBLIOGRAPHIE.}

[1] Barriol J. et Régnier J. - J. Physique Rad., i954, 15, 528 .

[2] Landolt-Bornstein. - 6 Auflage, I Band, 3 Teil, p. 5 ı 3.
[3] Pryce W. C. et Tuttle W. T. - Proc. Roy. Soc., i940, A 174, 207. 